\title{
Germanica
}

siècle

\section{Eyvind Johnson ou le bon usage de l'histoire}

Eyvind Johnson oder der gute Umgang mit Geschichte

\section{Philippe Bouquet}

\section{(2) OpenEdition}

\section{Journals}

Édition électronique

URL : http://journals.openedition.org/germanica/1261

DOI : 10.4000/germanica. 1261

ISSN : 2107-0784

Éditeur

Université de Lille

\section{Édition imprimée}

Date de publication : 1 décembre 1998

Pagination : 93-100

ISBN : 0984-2632F

ISSN : 0984-2632

\section{Référence électronique}

Philippe Bouquet, «Eyvind Johnson ou le bon usage de I'histoire », Germanica [En ligne], 23 | 1998, mis en ligne le 26 janvier 2012, consulté le 06 octobre 2020. URL : http://journals.openedition.org/ germanica/1261; DOI : https://doi.org/10.4000/germanica.1261

Ce document a été généré automatiquement le 6 octobre 2020.

(c) Tous droits réservés 


\title{
Eyvind Johnson ou le bon usage de l'histoire
}

\author{
Eyvind Johnson oder der gute Umgang mit Geschichte
}

Philippe Bouquet

Extrait d'une interview imaginaire :

2 Question: Comment se fait-il que, vous qui n'aimez pas le roman historique, ayez traduit deux romans ${ }^{1} \mathrm{~d}^{\prime} E y v i n d$ Johnson, le grand maitre du gerne en Suède ?

Réponse: C'est une excellente question et je vous remercie de me l'avoir posée (air connu). Il serait possible d'y répondre très simplement en disant que ce sont des textes remarquables, dus à l'un des grands écrivains de ce siècle. Mais ce ne serait pas amusant; je vais donc faire tout mon possible pour me comporter comme un universitaire (c'est vraiment pour moi un rôle de composition, mais je promets que ce sera la première et dernière fois) et tout compliquer à plaisir en disant que tous les romans de la fin de l'œuvre de Johnson possèdent un caractère historique, même quand ils n'en ont pas l'air, et que ceux qui se donnent pour tel le sont de façon assez paradoxale.

4 Soulignons d'abord un paradoxe dans la fortune littéraire de Johnson en France. Il se trouve, en effet, qu'il a eu le curieux privilège de voir l'un de ses premiers romans paraître en traduction française avant l'original suédois ${ }^{2}$. Cet insigne faveur n'a malheureusement pas été de bon augure et l'édition française a su, depuis, se venger de lui comme il le fallait. On peut affirmer sans crainte d'être démenti que Johnson est l'une des gloires mondiales les plus scandaleusement traitées par la France (et pourtant, la concurrence est rude sur ce plan). Non seulement l'essentiel de son œuvre, l'une des plus européennes qui soit, reste inconnu ici, mais lorsque les éditions Stock (c'était à la grande époque de Lucien Maury) ont eu la bonne idée de s'attaquer à son chef-d'œuvre, Le roman d'olofelles se sont arrêtées au premier volume (sur les quatre composant le roman dans sa totalité). Elles ont même ajouté l'insulte à la désinvolture puisque, lorsque Johnson a obtenu le prix Nobel en 1974, tout leur hommage a consisté à ressortir ce seul volume (jusque-là intitulé olof) sous le titre de Le roman d'olof qui 
était une escroquerie puisqu'il laissait supposer qu'il regroupait la totalité de l'œuvre et non pas le quart seulement. Depuis, il n'est paru que quatre volumes de lui (les deux derniers chez de petits éditeurs courageux) et l'on ignore toujours non seulement la suite du Romand'olof (qui est pourtant l'un des grands textes fondateurs du xx ${ }^{e}$ siècle, mais il est vrai qu'il illustre un genre, le roman prolétarien, qui n'a pas droit de cité dans notre république des lettres), mais aussi les romans « gidiens » des années 20 , les œuvres antinazies des années 30, la trilogie Krilon, véritable épopée de la démocratie au plus noir des années 40 , et les grandes œuvres historiques de la fin de sa vie. Chez nous, Johnson reste un iceberg dont on ne voit que la neuvième partie.

Dans les années 50, on a heureusement pu se faire une petite idée de ses dons dans le domaine historique grâce à deux publications un peu oubliées maintenant: Heureux Ulysse et De roses et de feu ${ }^{3}$.C'est une bonne base d'analyse. En effet, le premier adopte, a propos d'Ulysse, une démarche parallèle à celle de Joyce, mais de sens inverse en quelque sorte : au lieu de moderniser L'Odysséeen la situant dans le cadre de l'Irlande contemporaine, Johnson s'attache à nous faire comprendre la permanence du mythe en projetant sur lui, tel qu'il se présente originellement, la lumière des acquis récents dans le domaine des sciences humaines. C'est le comportement humain qui est sondé et non le cadre dans lequel se situe l'histoire. Cela vaut cependant au lecteur quelques surprises notoires. Disons que, de façon générale, le divin s'y réduit au quotidien (ce qui n'est peut-être pas très infidèle à la mythologie grecque, après tout, car les dieux s'y conduisaient souvent de façon fort humaine), mais l'héroïque aussi (ce qui l'est sans doute bien plus, en fait). La plupart des actes et des ruses y reçoivent des explications puisées dans le genre de motivations mises à nu par la psychologie moderne. L'un des exemples les plus intéressants est celui de Pénélope et de la fameuse tapisserie. Si la femme d'Ulysse défait la nuit ce qu'elle a fait le jour, ce n'est pas, nous fait comprendre Johnson, sous le coup d'une inexplicable lubie ou pour berner son monde. Non, Pénélope est simplement prise entre sa crainte de devoir opérer le choix tant redouté parmi les Prétendants et le désir de tout un chacun de voir son œuvre progresser. La piètre ruse d'Homère devient ici l'expression de l'ambiguïté fondamentale de l'être humain. Comme on le voit, l'historique s'efface ici devant l'analyse du comportement au point que l'on oublie vite que l'histoire se déroule quelques siècles avant notre ère. Les tenants du roman historique " en costumes ", avec reconstitution minutieuse (bien que souvent très imaginaire) de l'emploi du temps et du menu des personnages, risquent de ne pas y trouver leur compte.

Dans De roses et de feu,Johnson adopte une démarche assez analogue, qui nous incite sans cesse à lire les événements (rappelons qu'il s'agit de l'histoire des Possédées de Loudun) à la lumière de l'histoire contemporaine. Il ne modernise pas vraiment le cadre ni l'intrigue, mais procède par allusions à la politique (en donnant par exemple au couple Minet-Barrot une silhouette à la Goebbels), à la psychologie (notamment quant à la libido) et à la sociologie modernes (à propos des groupes de pression et du règne des absolus). Le tableau d'ensemble se trouve de ce fait dépouillé de presque tout pittoresque, mais cela ne fait que lui conférer une portée bien plus vaste quant à des phénomènes tels que l'intolérance et l'oppression. Le tableau est saisissant et le lecteur ne peut éviter de tirer les enseignements qui s'imposent quant à la période contemporaine et à sa propre vie : pensons ici au comportement de Drouin, qui incarne en quelque sorte le citoyen moyen de tous les temps, homme moyen sensuel, certes, mais aussi moyennement courageux, c'est-à-dire lâche. Le monde a toujours fourmillé de Drouin et en grouillera sans doute longtemps encore. Johnson, pour sa part, a 
compris qu'il faut parfois savoir mettre l'histoire entre parenthèses pour mieux en extraire la substantifique moelle.

7 Et c'est bien ce à quoi il s'attache dans les deux romans récemment parus en français, bien que de façon différente et, pour tout dire, complémentaire. Écartez le soleilse présente sous des dehors qui ont dû déconcerter la presse française, puisqu'elle s'est dans sa quasi-unanimité dispensée de commenter sa parution. C'est une sorte de jeu d'ombres sous la lumière des alpages. Dans ce chalet de montagne, qui s'avère bientôt être une scène de thêatre (All the world is a stage, disait déjà un certain Shakespeare), s'agitent un certain nombre de personnages, dont une sorte de révolutionnaire professionnel, Janus présenté tantôt sous le prénom de Frédéric, tantôt sous le nom de Gallo, trois femmes qui n'en font peut-être qu'une seule, au contraire, un contrebandier qui n'est pas forcément le plus mauvais de tous et un curieux personnage de spectateur-témoin-acteur (les trois rôles de l'être humain au regard de la dramaturgie). Tout ce petit monde cherche à échapper à un danger de nature politique qui les menace de deux côtés à la fois, et leur seule issue passe par un couloir d'avalanches où certains d'entre eux laisseront la vie. De prime abord, l'histoire paraît singulièrement absente et on peut être tenté de qualifier ce roman de tout sauf d'historique. Pourtant, la curieuse apparition de Churchill, seul personnage réel au milieu de toute cette fiction, pour incarner l'esprit de résistance (rappelons que le livre a été écriten 1951, mais qu'il a fallu attendre près d'un demi-siècle pour qu'il paraisse en France) nous incite ensuite à réviser notre jugement et à faire retour sur ce roman d'aventures, d'idées, d'amour, etc. (Denis Ballu, l'un des rares dans notre pays à s'être intéressés à ce livre parle de « roman du doute $»^{4}$ ) pour nous rendre compte qu'il nous parle au fond de notre Europe et de l'impossible neutralité face au mal et à une oppression qui ne cesse de renaître, même sous les pas des révolutionnaires les mieux intentionnés. Le curieux théâtre de l'action ne peut dès lors manquer de paraître symbolique, surtout quand on sait que Johnson a vécu plusieurs années en Suisse. Et ce lieu de neutralité qui se résume à une frontièren'est au fond que le fil d'un rasoir d'où il faut bien, tôt ou tard, basculer d'un côté ou de l'autre. Sous tous ses "peut-être ", ce roman est donc sans aucun doute un roman historique au plus haut niveau, un de ceux qui s'élèvent bien loin au-dessus d'elle pour en tirer les leçons et fournir aux hommes l'occasion d'une réflexion sur leur passé, certes, mais aussi et surtout sur leur avenir collectif. Car quelle peut être l'utilité de l'histoire, si ce n'est pour en tirer des enseignements qui se projettent fatalement dans le futur? Dans le cas contraire, elle se réduit à une variété bien vaine d'art pour l'art. Pour Johnson comme pour tous les grands historiens, le lieu véritable de l'histoire est le futur: le recul vers le passé n'est que l'élan nécessaire pour propulser la pensée humaine vers l'avant.

Dans Les nuages sur Métaponte,Johnson joue cette fois à fond la carte de l'historicité. Mais ne serait-ce pas pour mieux brouiller les cartes, une fois de plus? Le roman se déroule sur deux plans temporels: d'une part la Syracuse de l'Antiquité, à l'époque de Xénophon et de l'expédition des Dix mille, et d'autre part l'Europe des camps de concentration, ou est détenu J.-P. Lévy, archéologue qui a particulièrement étudié la Grande-Grèce. Le lien entre les deux est constitué par un élément postérieur, un Suédois du nom de Klemens Decorbie, qui a connu Lévy pendant la guerre et part sur ses traces sur les lieux mêmes de ses fouilles. Après l'épisode des Latomies de Syracuse, on ne tarde pas à comprendre que le thème profond du livre est la captivité sous ses deux visages, ancien et moderne, et que l'histoire, ici, n'est pas celle des manuels ou de ces romans auxquels «il ne manque pas un seul bouton de guêtres». Thémistogène, 
personnage historique peut-être fictif (ce qui fait de lui une sorte de héros de roman à l'envers) incarne en fait l'humanité pourchassée de tous les siècles. II se survit en Lévy, puis en Decorbie, et se survivra en d'autres hommes que l'histoire n'a pas encore appelés. Les enfants de Decorbie, surpris par l'histoire, ont pour toujours six et huit ans, comme le jour de leur mort dans les flots glacés de la mer du Nord, après le torpillage de leur navire. Les hommes sont pris dans cette immense masse temporelle qu'est l'histoire, passé, présent et avenir indissociables, fondus l'un dans l'autre. Quand cette force immense, puissante et subtile se déchaîne - ou fait seulement un caprice les hommes peuvent tout au plus faire le gros dos, comme sous l'orage. Mais elle est au fond telle les nuages qui passent sur Métaponte. Car si, même à la lecture de ce roman, elle peut nous donner l'impression d'un éternel recommencement, le livre est justement là pour nous convaincre du contraire. Les nuages sur Métaponte sont toujours semblables, ils ne sont jamais identiques.L'autonomie de l'événement existe bel et bien et l'homme doit y puiser des raisons d'espérer. Tel était d'ailleurs, le message que J.-P. Levy tentait de faire passer auprès de ses compagnons de captivité :

Parfois (murmura le professeur Levy), quand on est las, on peut être tenté de penser que tout est déjà arrivé dans tous les endroits ou l'on est passé. On peut penser que tout est terminé en tant qu'événement. On peut penser que tout est consommé. Mais ce n'est pas vrai, chers camarades. Chaque événement est parfaitement autonome. C'est pour cette raison qu'il y a encore de la vie. Et c'est ce qui fait que nous pouvons tous espérer ${ }^{5}$.

C'était aussi ce que disait déjà, en 1940, l'alter ego préféré de Johnson à l'époque, Marten Torpare :

C'est une force pour l'homme de pouvoir voir les choses historiquement, cela donne toujours, d'une façon ou d'une autre, des raisons d'espérer ${ }^{6}$.

On voit maintenant en quoi Johnson se distingue du romancier historique tel qu'on le conçoit en France, ou ce genre littéraire jouit d'une faveur supérieure à celle de tous les autres réunis, pour la seule raison qu'il permet de fuir le présent et, en fait, l'histoire, en la recouvrant de détails futiles qui la masquent, comme les arbres la forêt. Johnson ne vise à rien d'autre qu'à nous la rappeler sans cesse, au contraire, afin d'empêcher que l'on oublie ses leçons. Ce n'est pas pour rien qu'il se réfère à Gibbon, dans Ecartez le soleil.L'histoire doit être une constante mise en garde servant à dissuader l'humanité de commettre sans cesse les mêmes folies et les mêmes crimes. Cet espoir a certes été toujours floué, comme le démontre bien l'histoire humaine, justement. Mais, contrairement à tous ces écrivaillons bouffés de certitudes, Johnson veut nous enseigner le scepticisme et la vigilance. Pour lui, le roman historique ne peut donc être un but en soi, mais seulement " un raccourci vers une sorte de vérité sur soi-même, sur l'époque dans laquelle je vis ${ }^{7}$ ». D'où le sous-titre quelque peu provocant de Heureux Ulysse ("roman sur le temps présent»), et celui, plus audacieux encore, de Krilon: « roman sur le vraisemblable». Mais aucun autre n'aurait pu être plus juste et plus roboratif à la fois car, en ces années 1941 à 1943, Johnson ne tentait rien d'autre qu'une sorte d'histoire... du futur, celle de la survie de la démocratie. Sa conception du temps est globale. Dans Les nuages sur Métaponte, le temps n'est pas retrouvé,il est au contraire aboli.C'est l'homme qui est replacé au centre de cette masse temporelle, a charge pour lui de s'y orienter. S'il arrive donc à Johnson de s'attarder à évoquer pour nous tel ou tel événement du passé, c'est pour mieux nous projeter dans l'avenir, au prix d'une démarche véritablement dialectique, « un peu comme un homme à l'esprit historique, philosophique et pétri de culture peut voir la Rome, la Ninive ou la Gomorrhe 
modernes à travers des négatifs à demi transparents de la Rome, la Ninive ou la Gomorrhe passées, disparues, ensevelies sous la terre " fait-il dire à l'un de ses personnages ${ }^{8}$. Dans ces conditions, l'archéologie elle-même est essentiellement une méthode d'exploration de l'âme humaine et l'histoire rivalise au fond avec la conscience: "Dans la littérature, tu ne peux avoir la simultanéité, mais dans la conscience [...] dans la conscience, le passé et le présent sont là, l'un à côté de l'autre ", dit Crofter Brace?.

11 Avec Écartez le soleil,Johnson a mis la technique du nouveau roman au service d'une sorte de roman total, de roman collectif de l'humanité tout entière (au moins dans sa variante européenne). Il s'est apparemment écarté de tout point de vue historique pour mieux nous donner une leçon d'histoire. Dans Les nuages sur Métaponte, il a procédé de façon inverse: il s'est enfoncé dans l'historique jusqu'au cou pour mieux nous en montrer la relativité et nous inciter à le dominer. Une telle conception de l'histoire allie ce que la littérature prolétarienne peut nous apporter de meilleur (ce «courage » que Crofter Brace nous souhaite au début comme à la fin d'Ecartez le soleil), aux enseignements de l'humanisme traditionnel. C'est évidemment beaucoup pour les petits-maîtres de la presse et du monde culturel parisiens, trop occupés à surveiller leur lookdans le fenestron. Ils risqueraient une fatale indigestion, s'ils ouvraient des livres de ce genre.

L'INTERVIEWER : Je vous remercie de toutes ces explications. Malheureusement, il ne sera peut-être pas possible de les publier. Vous vous êtes égaré dans des considérations qui n'ont rien à faire dans une revue scientifique et notre comité de lecture est très strict sur ce point. C'est dommage, car vous êtes l'un des premiers à répondre à notre appel.

L'INTERVIEWÉ: Oh, il suffira de faire semblant d'avoir égaré vos notes. Cela n'aura rien pour me surprendre. Je n'ai pas oublié les enseignements de mon histoire personnelle. Mais il m'intéresse de voir si elle se répétera vraiment ou si Johnson a raison quant à «l'autonomie de l'événement", même en ce qui concerne ce tout petit bout de la lorgnette que je représente.

\section{NOTES}

1. Écartez le soleil, éditions Manya, 1992, 357 pages, et Les nuages sur Métaponte, éditions Esprit ouvert, 1995, 340 pages.

2. Il s'agit de Lettre recommandée, paru dès 1927 aux éditions Kra et seulement en 1928, sous le titre Stad i ljus, en Suède.

3. Respectivement : Gallimard, 1950 et Stock, 1956.

4. Nouvelles du Nord, nol, Nantes, 1993, p. 20.

5. Écartez le soleil, p. 261.

6. Soldatens återkomst, p. 266.

7. Röster i radio, $1960, \mathrm{n}^{\circ} 52$, p. 14.

8. Krilons resa, p. 84.

9. Lägg undan solen, p. 55. 


\section{RÉSUMÉS}

L'article se propose de décrire la vision de l'histoire d'E. Johnson telle qu'elle apparaît à l'analyse de ses romans. Tout d'abord, c'est le comportement humain qui est sondé et non le cadre dans lequel se situe l'histoire, de sorte que l'historique s'efface devant l'analyse du comportement. L'auteur ne modernise pas le cadre ni l'intrigue mais procède par allusions à la politique. Le tableau d'ensemble se trouve de ce fait dépouillé de presque tout pittoresque, mais cela ne fait que lui conférer une portée plus vaste. Pour Johnson, le lieu véritable de l'histoire est le futur : le recul vers le passé n'est que l'élan nécessaire pour propulser la pensée humaine vers l'avant. Les hommes sont pris dans l'immense masse temporelle qu'est l'histoire, passé, présent et avenir indissociables, fondus l'un dans l'autre. L'être humain est replacé au centre de cette masse temporelle, à charge pour lui de s'y orienter, car l'autonomie de l'événement existe bel et bien, et l'homme doit y puiser des raisons d'espérer. L'histoire étant une constante mise en garde, le roman historique ne peut être un but en soi, mais seulement « un raccourci vers une sorte de vérité sur soi-même, sur l'époque dans laquelle je vis ».

Der Artikel beabsichtigt, Eyvind Johnsons Vision von Geschichte zu beschreiben, so wie sie bei der Analyse seiner Romane erscheint. Zuallererst wird das menschliche Verhalten erforscht und nicht der Rahmen, in dem die Geschichte situiert ist, so daß das Historische hinter die Analyse des Verhaltens in den Hintergrund tritt. Der Autor paßt weder den Rahmen noch die Handlungen der neuen Zeit an, sondern arbeitet mit Anspielungen an die Politik. Deshalb wird in den Romanen alles Malerisch-Pittoreske ausgespart, doch verleiht ihnen das nur eine größere Tragweite. Für Johnson ist der echte Ort der Geschichte die Zukunft: Der Rückzug in die Vergangenheit ist nur der notwendige Schwung, um das menschliche Denken voranzutreiben. Die Menschen sind in der riesigen zeitlichen Masse der Geschichte gefangen, Vergangenheit, Gegenwart und Zukunft sind untrennbar ineinander verschmolzen. Der Mensch wird ins Zentrum dieser zeitlichen Masse gestellt, um sich dort zu orientieren, denn die Eigenständigkeit des Ereignisses existiert sehr wohl und daraus muß der Mensch Hoffnung schöpfen. Da die Geschichte eine ständige Warnung ist, kann der historische Roman kein Ziel an sich sein, sondern nur « eine Abkürzung zu einer Art Wahrheit über sich selbst und über die Epoche, in der ich lebe ».

\section{AUTEUR}

PHILIPPE BOUQUET

Université de Caen 